\title{
Topological Stabilization and Dynamics of Self-Propelling Nematic Shells
}

\author{
Babak Vajdi Hokmabad $\odot,{ }^{1,2,}$ Kyle A. Baldwin $\odot,{ }^{1,3,{ }^{*}}$ Carsten Krüger, ${ }^{1}$ Christian Bahr $\odot,{ }^{1}$ and Corinna C. Maass $\odot^{1, \dagger}$ \\ ${ }^{1}$ Max Planck Institute for Dynamics and Self-Organization, Am Faßberg 17, 37077 Göttingen, Germany \\ ${ }^{2}$ Institute for the Dynamics of Complex Systems, Georg August Universität, 37077 Göttingen, Germany \\ ${ }^{3}$ SOFT Group, School of Science and Technology, Nottingham Trent University, Nottingham NG11 8NS, United Kingdom
}

(Received 4 July 2019; published 23 October 2019)

\begin{abstract}
Liquid shells (e.g., double emulsions, vesicles, etc.) are susceptible to interfacial instability and rupturing when driven out of mechanical equilibrium. This poses a significant challenge for the design of liquidshell-based micromachines, where the goal is to maintain stability and dynamical control in combination with motility. Here, we present our solution to this problem with controllable self-propelling liquid shells, which we have stabilized using the soft topological constraints imposed by a nematogen oil. We demonstrate, through experiments and simulations, that anisotropic elasticity can counterbalance the destabilizing effect of viscous drag induced by shell motility and inhibit rupturing. We analyze their propulsion dynamics and identify a peculiar meandering behavior driven by a combination of topological and chemical spontaneously broken symmetries. Based on our understanding of these symmetry breaking mechanisms, we provide routes to control shell motion via topology, chemical signaling, and hydrodynamic interactions.
\end{abstract}

DOI: 10.1103/PhysRevLett.123.178003

The capability to produce controllable, actively selfpropelling microcapsules would present a leap forward in the development of artificial cells, microreactors, and microsensors. Inactive microcapsules have been developed in the form of double emulsions (droplet shells), which have been applied as, e.g., reactive microcontainers [1], synthetic cell membranes [2], food and drug capsules [3,4], optical devices [5-7], and biotic sensors [8]. However, these highly structured compound droplets are usually nonmotile, and any actuation that displaces their liquid cores makes them susceptible to shell rupture if the interfaces of the nested compartments can coalesce. Alternative compartmentalized structures such as vesicles, capsids, or polymersomes typically possess immobile interfaces that impede self-actuation. Hence, engineering such motile systems requires further complexities in design and fabrication [9-11]. In contrast, to survive motility, any liquid shell with mobile interfaces requires a stabilizing force to counter the destabilizing swimming dynamics.

In this Letter, we present a new approach to the problem of combining encapsulation with autonomous motility, by using nematic active double emulsions, where anisotropic micellar solubilization induces motility, and the nematoelasticity of the shell provides stability without requiring

Published by the American Physical Society under the terms of the Creative Commons Attribution 4.0 International license. Further distribution of this work must maintain attribution to the author(s) and the published article's title, journal citation, and DOI. further complexities in the design. Through experiments and simulation of the elastic energy in the liquid crystal shell, we show that active shells are stable only in the nematic state. We demonstrate that the shell dynamics are dictated by anisotropic self-generated chemical fields, broken topological symmetries, and hydrodynamic interactions, and that by tuning these factors we can control and direct their motion, providing avenues for applications in transport, guidance, and targeted release. Our framework provides a bottom-up approach for developing functional micromachines using established physicochemical mechanisms.

Our active double emulsion system is comprised of water-in-oil-in-water droplet shells. Shells self-propel while slowly dissolving in a micellar surfactant solution.

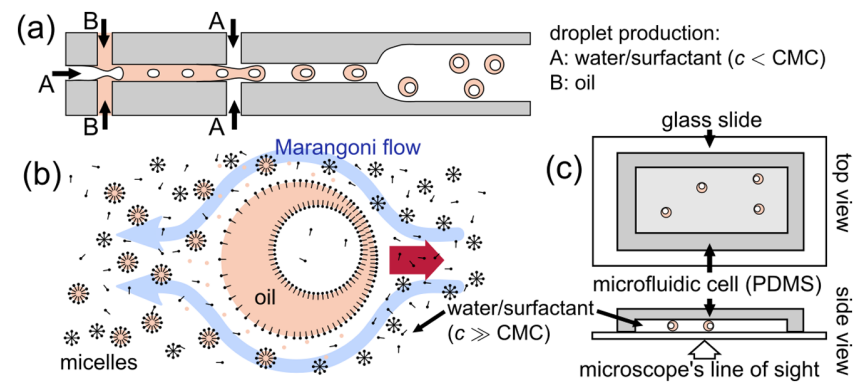

FIG. 1. Droplet production and setup schematics. (a) Double emulsion production via microfluidic flow junctions. (b) Droplet propulsion via a self-sustaining Marangoni gradient in the interface. (c) Microfluidic cell and inverted microscope setup for quasi-2D observation. All schematics are not to scale; spherical shells and micelles are represented by their $2 \mathrm{D}$ cross section. 
Micelles swell while filling with oil, which depletes the surfactant coverage of the shell's posterior. This induces a self-sustaining tension gradient in the external oil-water interface that drives the droplet motion [12-14] [Fig. 1(b)]. Swimming droplets shed persistent trails of oil-filled micelles, from which they are subsequently repelled [15].

We use the nematogen $5 \mathrm{CB}$ as the oil phase and solutions of the anionic surfactant TTAB as the aqueous phases, where the internal core droplet is submicellar $(c=0.75 \mathrm{CMC})$ and the external swimming medium is supramicellar $(c>30 \mathrm{CMC})$. We mass produce highly monodisperse oil droplet shells using consecutive microfluidic cross junctions in a flow-focusing configuration [2,16] [Fig. 1(a)] and observe them in quasi-2D microfluidic cells under videomicroscopy [Fig. 1(c)].

Despite the displacement of the aqueous core towards the shell boundary [Fig. 2(a)], the shells self-propel stably and reproducibly for long times, dissolving down to thin shells with a minimum stable shell/core radii fraction of $R_{s} / R_{c} \approx 1.05$. The life stages of these self-propelling shells [Figs. 2(b) and 2(c)] fall into three regimes. (I) "Shark-fin" meandering: At early times, the core is small compared to the shell diameter [Fig. 2(b), top], and it is deflected considerably from the polar axis of the shell, resulting in a meandering instability. (II) Thin shells: As the shell thins, eventually there is little room for significant asymmetry in the shell-core arrangement [Fig. 2(b), bottom]. During this stage, the motion grows noisy while the speed decreases, until propulsion stops. (III) Single emulsion: On reaching a critical minimum thickness, the shell bursts, reconstituting into a single oil droplet. From a comparison of preburst and
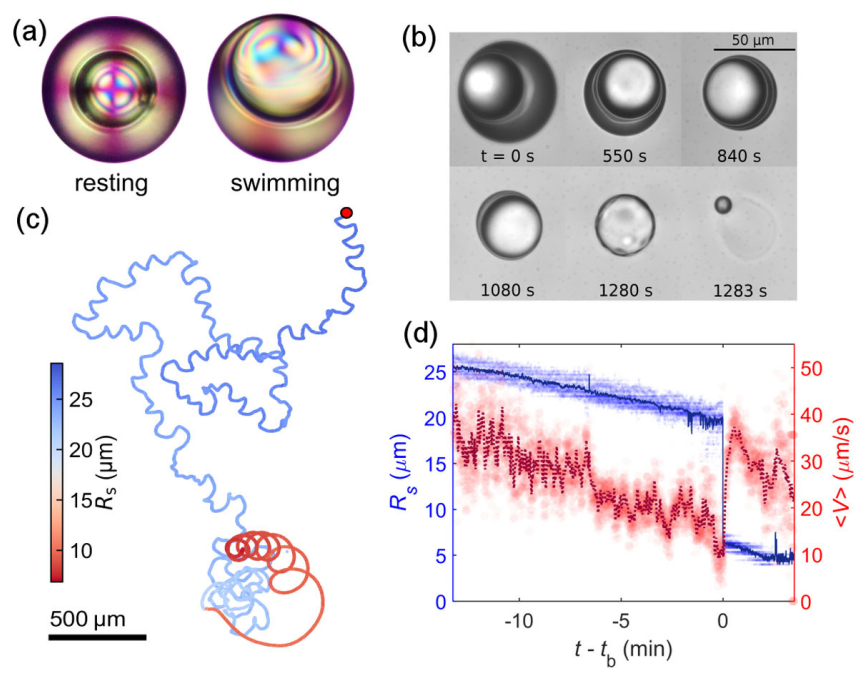

FIG. 2. Life stages of active shells. (a) Polarized images of resting and swimming shells. (b) Video stills of droplet life stages. (c) Example trajectory colored by shell radius $R_{s}$, recorded over $40 \mathrm{~min}$. The sudden change of color from blue to red corresponds to the burst moment. (d) Average speed $V$ (dotted, red) and radius $R_{s}$ (solid, blue) for 13 shells, with time $t$ relative to bursting time $t_{b}$ (scatter plots: values for all experiments). postburst radii, we estimate that the average shell thickness at this point is less than $1 \mu \mathrm{m}$ [Fig. 2(d)]. The droplet then propels with an undisturbed internal convection, leading to a sudden increase in speed [Fig. 2(d)] and a curling motion as observed in nematic single emulsions [25] [Fig. 2(c)].

In contrast to these reproducible stages in nematic shells, we find that under otherwise identical conditions, shells made from isotropic oils (CB15 or 5CB/BPD, see Ref. [16]) burst significantly earlier. Figure 3(a) shows burst statistics for $5 \mathrm{CB}$ shells, where below the clearing point ( $T<34.5^{\circ} \mathrm{C}$, nematic), shells survive for long times, whereas above the clearing point $\left(T>34.5^{\circ} \mathrm{C}\right.$, isotropic), most droplets do not reach the thin shell stage.

We attribute the shell stability to a nemato-elastic energy barrier: $5 \mathrm{CB}$ molecules arrange to minimize the elastic energy associated with the deviations from a uniform director field imposed by the boundary conditions (here, homeotropic anchoring [26]). In a resting shell, this causes a radially symmetric arrangement of the director field [27] with the aqueous core at the center. In a moving shell, the internal flow drives the core off center; the director field is therefore distorted both by the displacement of the core and the flow field, such that the stored elastic energy is increased.

To estimate the competing forces, we numerically simulate the director field inside the shell and calculate the elastic energy $E$ stored in a resting shell with a core displaced by a distance $d$. We apply a common numeric minimization technique [22,23] based on the $\mathbf{Q}$ tensor representation [24] of the nematic director field [16]. The tensor elements of a uniaxial nematic with scalar order parameter $S$ and local director $\mathbf{n}$ are given by

$$
Q_{j k}=\frac{S}{2}\left(3 \mathbf{n}_{j} \mathbf{n}_{k}-\delta_{j k}\right) .
$$

Since topological defects are not present in the director field of our shells, we neglect a variation of the magnitude of $S$ and assume a constant value $S=1$. For the calculation of the elastic energy density $f_{e}$, we use the one-constant approximation of the nematic elasticity, i.e., $K_{\text {splay }}=$ $K_{\text {twist }}=K_{\text {bend }}=K$. Then, $f_{e}$ is obtained as

$$
f_{e}=\frac{K}{9} Q_{j k, l} Q_{j k, l},
$$

where $Q_{j k, l}=\partial_{l} Q_{j k}$. The total elastic energy $E$ is then calculated by integration over the shell volume $\Omega$ :

$$
E=\int_{\Omega} f_{e} \mathrm{~d} \Omega
$$

As shown in Fig. 3(b), we find that $E$ increases by a factor of $E_{i} / E_{c} \approx 1.4$ when the core droplet is located at the outer interface $\left(E=E_{i}, d=d_{\max }\right)$, as compared to the centered configuration $\left(E=E_{c}\right)$. Remarkably, we find only a minor dependence on the thickness of the nematic shell. Note that 

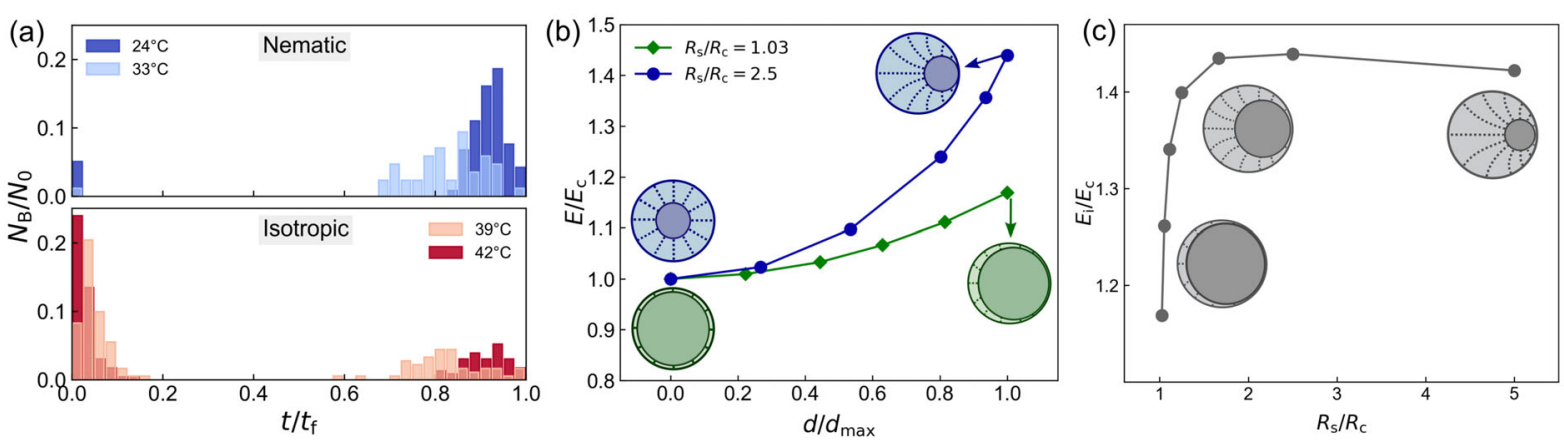

FIG. 3. Stabilization of the active shells. (a) Burst statistics (number of bursts $N_{B}$ normalized to initial number of shells $N_{0}$ ) for shells below and above the clearing point, plotted against time $t$ normalized to final time $t_{f}$ with no remaining shells $\left[t_{f}\left(24{ }^{\circ} \mathrm{C}\right) \approx 45\right.$ min, $t_{f}\left(39^{\circ} \mathrm{C}\right) \approx 10 \mathrm{~min}$, initial $\left.R_{s}=30 \mu \mathrm{m}, R_{c}=18 \mu \mathrm{m}\right]$. (b) Elastic energy $E / E_{c}\left(E_{c}\right.$ : core at center of droplet) as a function of core displacement $d / d_{\max }$, with $d_{\max }=R_{s}-R_{c}-100 \mathrm{~nm}$, for two different ratios $R_{s} / R_{c}$. (c) Ratio of elastic energies $E_{i} / E_{c}\left(E_{i}\right.$ : core droplet close to outer shell interface) against ratio of radii $R_{s} / R_{c}$, using $R_{c}=25 \mu \mathrm{m}$ and $R_{s}$ shrinking from $125 \mu \mathrm{m}$ to $25.6 \mu \mathrm{m}$. Schematics are illustrative, and illustrated radius values do not directly correspond to the simulation parameters.

$E_{i} / E_{c}$ drops significantly towards unity only for $R_{s} / R_{c}<$ 1.1 [Fig. 3(c)]; i.e., the elastic energy barrier vanishes only in the limit of zero shell thickness.

We calculate the elastic force $F_{e}=\partial E / \partial d$ acting on a core that has been displaced to the boundary of a $5 \mathrm{CB}$ shell $[28,29]$ to be of the order of $\approx 100 \mathrm{pN}$. This is equivalent to the Stokes drag [12],

$$
F_{S}=2 \pi R_{c} v \frac{2 \eta_{5 \mathrm{CB}}+3 \eta_{\mathrm{aq}}}{1+\eta_{\mathrm{aq}} / \eta_{5 \mathrm{CB}}},
$$

acting on an aqueous core moving through bulk $5 \mathrm{CB}$ at $v \approx 6 \mu \mathrm{m} / \mathrm{s}$, which is comparable to the velocity of the convective flow in our shells. We propose that the

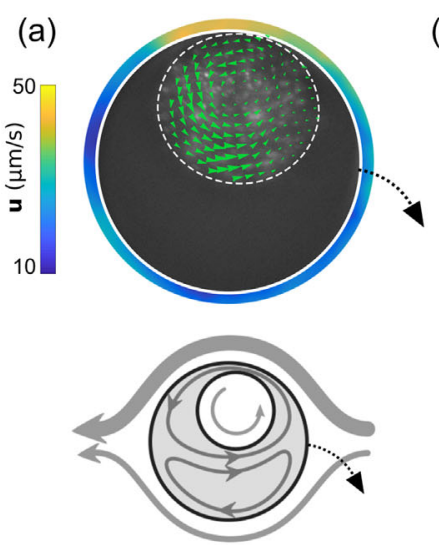

(d) No core; Radial hedgehog defect

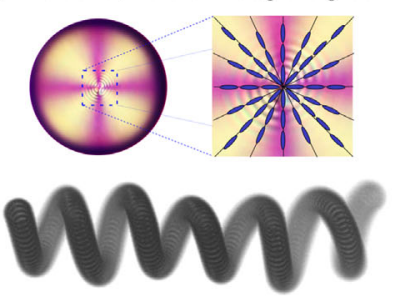

(b) rurrorsa

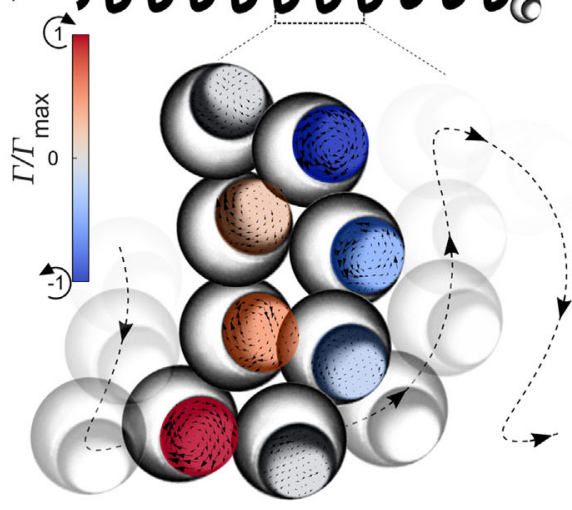

(e) One core; No defect

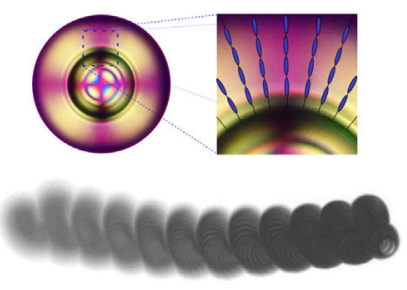

(c)
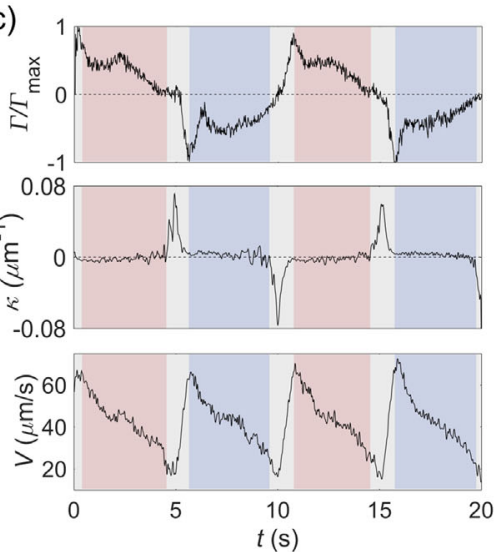

(f) Two cores; Hyperbolic hedgehog defect

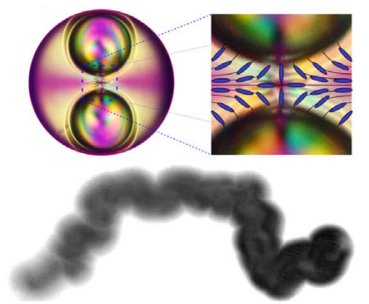

FIG. 4. Swimming behavior. (a) Top: PIV data for flow at the outer interface and in the core. Bottom: Schematic of core and flow arrangement. (b) Top: Shark-fin meandering of swimming trajectory over 2 minutes $\left(R_{s}=36 \mu \mathrm{m}\right)$; the shell switches periodically between clockwise and anticlockwise turns. Bottom: Zoomed-in view of the shell-core alignment with the swimming trajectory, with superimposed core flow fields and color-coded circulation. (c) Core circulation $\Gamma / \Gamma_{\max }$, local curvature $\kappa$, and shell speed $V$ vs time over four meandering periods. (d)-(f) Nematic structure (top, polarized images of the droplets at rest with overlaid director field schematic) and 3D swimming trajectories (bottom, multiple exposure micrograph captured over $60 \mathrm{~s}$ ) for a no-core droplet (d), a single-core droplet (e), and a two-core droplet (f). See Movie S8 in Ref. [16]. 
nemato-elastic repulsion provides a significant, although not insurmountable, barrier against coalescence.

We analyze the meandering dynamics by simultaneously tracking the circulation of the flow inside the core $\Gamma(t)$, local trajectory curvature $\kappa(t)$, and propulsion speed $V(t)$ [Figs. 4(a)-4(c)]. In quasi-2D confinement, the core is trapped off axis inside the convective torus, where it corotates with the convective flow, as shown by the core flow and color-coded $\Gamma$ values in Figs. 4(a) and 4(b). In this arrangement, there is less viscous resistance to the driving interfacial flows in the part of the shell containing the core, resulting in asymmetric flow with respect to the direction of motion [shown by the color bar in Fig. 4(a)] and a curved trajectory. This eventually curves the shell back towards its own trail, where chemotactic repulsion causes $V$ and $\Gamma$ to slowly decay and then abruptly reverse - the tip of the shark-fin motion [Figs. 4(b) and 4(c)]. This abrupt reorientation corresponds to a spike in the local curvature and is followed by a sharp acceleration [Fig. 4(c)], caused by repulsion from the local gradient of filled micelles. Because of the flow reversal, in the comoving reference frame, the core has now switched sides, and the shell curves in the opposite direction, once again towards its own trail. We distinguish three timescales: a short timescale $(\approx 1 \mathrm{~s})$ for autochemotactically driven abrupt reorientation, an intermediate timescale $(\approx 5 \mathrm{~s})$ for the curved motion between two shark-fin tips, and a long timescale (>100 s) corresponding to the persistent motion imposed by the chemical field in the trail of the shell (cf. Ref. [16], Fig. S2).

To further investigate the role of the core in breaking the flow symmetry, we have additionally experimented in 3D bulk media, using deep microfluidic wells and matching the density of oil and swimming media by substituting a fraction of water with deuterated water in the surfactant solution. We compare the dynamics of droplets with zero, one, and two cores [Figs. 4(d)-4(f)]. With no core, we reproduce previous findings [25], where the displacement of the radial "hedgehog" defect induces a torque on the droplet. Given the freedom of a third dimension, the droplet is not arrested by its own trail and does not reverse its direction, resulting in helical trajectories. With one core, we observe similar behavior, with the core precessing around the axis of motion. Shells propel in more tightly wound helices than single emulsions, which can be understood in terms of the torque applied by the respective viscous anisotropy: For shells, it is the viscosity ratio of oil and water, $\eta_{2}(5 \mathrm{CB}) / \eta\left(\mathrm{H}_{2} \mathrm{O}\right) \approx 50$; in contrast, for single emulsions [25], it refers to the intrinsic viscous anisotropy of a nematic liquid crystal $\eta_{2}(5 \mathrm{CB}) / \eta_{\text {iso }}(5 \mathrm{CB}) \approx 3$ [29]. With two cores, this broken symmetry argument does not hold, and thus we are able to rectify the meandering motion.

While a single-core nematic shell is defect free and spherically symmetric at rest, double-core shells have a fixed axis set by the two cores, with a topological charge of +1 resolved by a hyperbolic hedgehog defect or a defect (a)
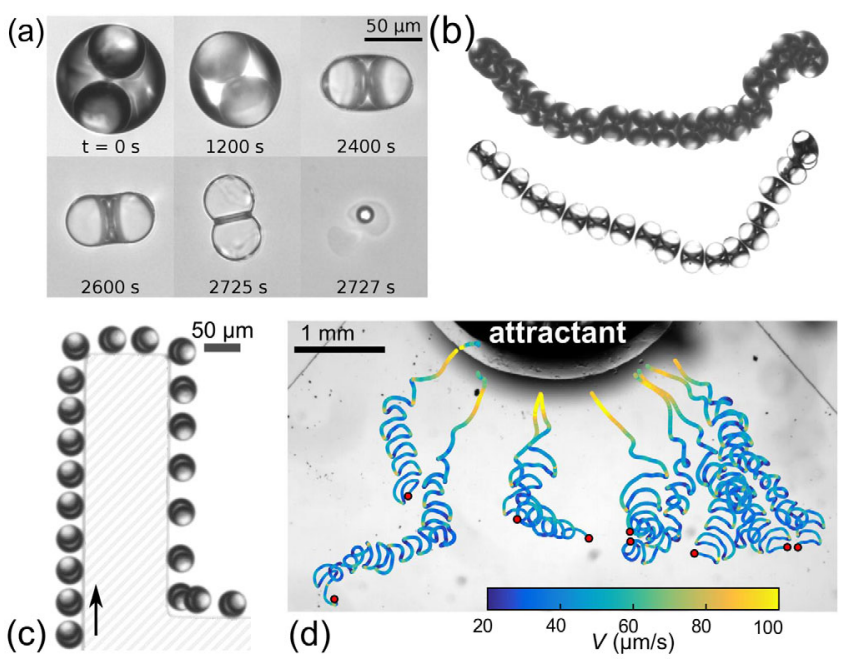

FIG. 5. Control of shell dynamics. Micrographs of shells with two cores, showing (a) long-time stability and (b) no meandering in 2D confinement (multiple exposure micrographs at two shell thicknesses). Because of the larger initial volume of the oil phase, survival times are increased compared to Fig. 2(b). (c) Topographical guidance by walls. Multiple exposure micrograph taken over 65 seconds at 3 second intervals. (d) Chemotaxis: Diffusing surfactant guides shells to the left (trajectories colored by shell speed $V$ ).

loop [30-32]. This defect provides a barrier against core coalescence [33]. Hence, as in the single-core case, the shell thickness shrinks to $\approx 1 \mu \mathrm{m}$ until the shell bursts [Fig. 5(a)]. The most likely flow field configuration inside a moving double-core shell is with both cores trapped on opposite sides of the convection torus and no symmetry breaking mechanism or curling. Instead, the shell moves perpendicularly to the core alignment, with some rotational fluctuations [demonstrated in quasi-2D, Fig. 5(b)].

Changing the topology of the liquid crystal, e.g., by controlling the number of cores, provides one method to rectify the propulsion dynamics. However, based on our work on single emulsions [15,34], we have further options to guide self-propelling shells and improve their utility as cargo carrying vessels and sensors, by exploiting microfluidic topography $[35,36]$ and chemical gradients. First, we have topographical guidance: Fig. 5(c) shows a shell swimming along a wall, turning both convex corners without detachment and concave corners without arrest (further examples are given in Fig. S4 [16]). Second, we have chemotactic guidance: In Fig. 5(d), crystalline surfactant ("attractant") is allowed to dissolve into a quasi-2D cell. The resulting gradient extends $\approx 1 \mathrm{~mm}$ into the cell, attracting the shells, doubling their speed, and rectifying the meandering instability.

In conclusion, we have developed a versatile platform for microscopic cargo delivery: self-propelling droplet shells. While motility induces convection that acts to destabilize these cargo vessels, we have demonstrated through experiments and simulations that nemato-elasticity 
can be employed as a topologically stabilizing agent, a fact we anticipate will be utilized in novel designs of microreactors and artificial cells. We have also provided pathways for guiding the trajectories of these droplets, through both chemical signaling and topography. Finally, we have analyzed the interesting swimming behavior of these selfpropelling shells, and we anticipate that the understanding of the rich shark-fin meandering dynamics will impact the design of artificial microswimmers, where swimming behavior can be tweaked by tuning the routes for spontaneous symmetry breaking.

Financial support from the Deutsche Forschungsgemeinschaft (SPP1726 Microswimmers) is gratefully acknowledged. We thank Stephan Herminghaus for stimulating discussions and Julien Petit for invaluable experimental advice.

*These authors contributed equally to this work. corinna.maass@ds.mpg.de

[1] E. Lorenceau, A. S. Utada, D. R. Link, G. Cristobal, M. Joanicot, and D. A. Weitz, Langmuir 21, 9183 (2005).

[2] J. Petit, I. Polenz, J.-C. Baret, S. Herminghaus, and O. Bäumchen, Eur. Phys. J. E 39, 59 (2016).

[3] M. A. Augustin and Y. Hemar, Chem. Soc. Rev. 38, 902 (2009).

[4] S.-H. Kim, J. W. Kim, J.-C. Cho, and D. A. Weitz, Lab Chip 11, 3162 (2011).

[5] L. Chen, Y. Li, J. Fan, H. K. Bisoyi, D. A. Weitz, and Q. Li, Adv. Opt. Mater. 2, 845 (2014).

[6] S. Nagelberg, L. D. Zarzar, N. Nicolas, K. Subramanian, J. A. Kalow, V. Sresht, D. Blankschtein, G. Barbastathis, M. Kreysing, T. M. Swager, and M. Kolle, Nat. Commun. 8, 14673 (2017).

[7] L. D. Zarzar, V. Sresht, E. M. Sletten, J. A. Kalow, D. Blankschtein, and T. M. Swager, Nature (London) 518, 520 (2015).

[8] Q. Zhang, S. Savagatrup, P. Kaplonek, P. H. Seeberger, and T. M. Swager, ACS Cent. Sci. 3, 309 (2017).

[9] B. V. V. S. P. Kumar, A. J. Patil, and S. Mann, Nat. Chem. 10, 1154 (2018).

[10] A. V. Singh, Z. Hosseinidoust, B.-W. Park, O. Yasa, and M. Sitti, ACS Nano 11, 9759 (2017).

[11] A. Joseph, C. Contini, D. Cecchin, S. Nyberg, L. RuizPerez, J. Gaitzsch, G. Fullstone, X. Tian, J. Azizi, J. Preston, G. Volpe, and G. Battaglia, Sci. Adv. 3, e1700362 (2017).
[12] S. Herminghaus, C. C. Maass, C. Krüger, S. Thutupalli, L. Goehring, and C. Bahr, Soft Matter 10, 7008 (2014).

[13] C. C. Maass, C. Krüger, S. Herminghaus, and C. Bahr, Annu. Rev. Condens. Matter Phys. 7, 171 (2016).

[14] Z. Izri, M. N. van der Linden, S. Michelin, and O. Dauchot, Phys. Rev. Lett. 113, 248302 (2014).

[15] C. Jin, C. Krüger, and C. C. Maass, Proc. Natl. Acad. Sci. U.S.A. 114, 5089 (2017).

[16] See Supplemental Material at http://link.aps.org/ supplemental/10.1103/PhysRevLett.123.178003 for further details on emulsion fabrication, observation, and numerical simulation techniques, which includes citations [17-24].

[17] D. Qin, Y. Xia, and G. M. Whitesides, Nat. Protoc. 5, 491 (2010).

[18] A. Schmit, L. Salkin, L. Courbin, and P. Panizza, Soft Matter 10, 4743 (2014).

[19] J. C. Crocker and D. G. Grier, J. Colloid Interface Sci. 179, 298 (1996).

[20] W. Thielicke and E. Stamhuis, J. Open Res. Softw. 2, e30 (2014).

[21] H. Wioland, F. G. Woodhouse, J. Dunkel, and R. E. Goldstein, Nat. Phys. 12, 341 (2016).

[22] H. Mori, J. Eugene C. Gartland, J. R. Kelly, and P. J. Bos, Jpn. J. Appl. Phys. 38, 135 (1999).

[23] M. Ravnik and S. Žumer, Liq. Cryst. 36, 1201 (2009).

[24] P.-G. De Gennes, Mol. Cryst. Liq. Cryst. 12, 193 (1971).

[25] C. Krüger, G. Klös, C. Bahr, and C. C. Maass, Phys. Rev. Lett. 117, 048003 (2016).

[26] J. M. Brake, A. D. Mezera, and N. L. Abbott, Langmuir 19, 6436 (2003).

[27] T. Lopez-Leon and A. Fernandez-Nieves, Colloid Polym. Sci. 289, 345 (2011).

[28] P. P. Karat and N. V. Madhusudana, Mol. Cryst. Liq. Cryst. 40, 239 (1977).

[29] A. G. Chmielewski, Mol. Cryst. Liq. Cryst. 132, 339 (1986).

[30] N. D. Mermin, Rev. Mod. Phys. 51, 591 (1979).

[31] T. C. Lubensky, D. Pettey, N. Currier, and H. Stark, Phys. Rev. E 57, 610 (1998).

[32] P. Poulin and D. A. Weitz, Phys. Rev. E 57, 626 (1998).

[33] P. Poulin, H. Stark, T. C. Lubensky, and D. A. Weitz, Science 275, 1770 (1997).

[34] C. Jin, B. Vajdi Hokmabad, K. A. Baldwin, and C. C. Maass, J. Phys. Condens. Matter 30, 054003 (2018).

[35] S. Das, A. Garg, A. I. Campbell, J. Howse, A. Sen, D. Velegol, R. Golestanian, and S. J. Ebbens, Nat. Commun. 6, 8999 (2015).

[36] J. Simmchen, J. Katuri, W. E. Uspal, M. N. Popescu, M. Tasinkevych, and S. Sánchez, Nat. Commun. 7, 10598 (2016). 